\title{
Combat-Related Intradural Gunshot Wound to the Thoracic Spine: Significant Improvement and Neurologic Recovery Following Bullet Removal
}

\author{
Thijs M Louwes ${ }^{1}$, William H Ward ${ }^{2}$, Kendall H Lee ${ }^{1}$, Brett A Freedman ${ }^{3}$ \\ ${ }^{1}$ Department of Neurologic Surgery, Mayo Clinic, Rochester, MN, USA \\ ${ }^{2}$ Department of General Surgery, Naval Medical Center Portsmouth, Portsmouth, VA, USA \\ ${ }^{3}$ Department of Orthopedic Surgery, Landstuhl Regional Medical Center, Landstuhl, Germany
}

The vast majority of combat-related penetrating spinal injuries from gunshot wounds result in severe or complete neurological deficit. Treatment is based on neurological status, the presence of cerebrospinal fluid (CSF) fistulas, and local effects of any retained fragment(s). We present a case of a 46-year-old male who sustained a spinal gunshot injury from a 7.62-mm AK-47 round that became lodged within the subarachnoid space at T9-T10. He immediately suffered complete motor and sensory loss. By 24-48 hours postinjury, he had recovered lower extremity motor function fully but continued to have severe sensory loss (posterior cord syndrome). On post-injury day 2, he was evacuated from the combat theater and underwent a T9 laminectomy, extraction of the bullet, and dural laceration repair. At surgery, the traumatic durotomy was widened and the bullet, which was laying on the dorsal surface of the spinal cord, was removed. The dura was closed in a water-tight fashion and fibrin glue was applied. Postoperatively, the patient made a significant but incomplete neurological recovery. His stocking-pattern numbness and sub-umbilical searing dysthesia improved. The spinal canal was clear of the foreign body and he had no persistent CSF leak. Postoperative magnetic resonance imaging of the spine revealed contusion of the spinal cord at the T9 level. Early removal of an intra-canicular bullet in the setting of an incomplete spinal cord injury can lead to significant neurological recovery following even high-velocity and/or high-caliber gunshot wounds. However, this case does not speak to, and prior experience does not demonstrate, significant neurological benefit in the setting of a complete injury.

Keywords: Gunshot wound; Foreign body; Spinal cord injury; Laminectomy; Recovery of function

\section{Introduction}

While the majority of combat-related injuries among US military service members in Iraq and Afghanistan have been caused by explosive devices, high-velocity gunshot wounds (GSWs) are still present in $20 \%$ of casualties [1]. Unfortunately, these devastating injuries are not confined to the combat setting, with ever-increasing rates of GSWs, particularly from high- velocity, high-caliber rounds, occurring in the civilian sector too. Here, we present a rare case of a military-grade assault weapon gunshot to the thoracic spinal column, sustained in combat, with spontaneous recovery of motor function within 24-48 hours and significant, but incomplete, improvement of posterior cord syndrome, the rarest of the incomplete spinal cord syndromes, after surgical removal of the bullet fragment.

Received Feb 19, 2014; Revised Feb 19, 2014; Accepted Feb 25, 2014

Corresponding author: Brett A Freedman

Landstuhl Regional Medical Center, CMR 402, PO Box 1503, APO, AE 09180, Germany

Tel: +49-6371865188, Fax: +49-6371865949, E-mail: brett.a.freedman.mil@mail.mil 
There have been previous cases of significant neurological recovery after penetrating spinal injuries, although typically at the conus and cauda equina level, with lower velocity bullets and/or with different injury mechanism [2-4]. However, we believe that this is the first report of a highvelocity, high-caliber GSW associated with immediate complete neurological deficits, being treated with timely removal of the fragment and resulting significant neurological recovery, as well as obviation of the potential pitfalls of a retained free-floating intra-canicular foreign body.

\section{Case Report}

The patient was a 46-year-old white male, who sustained a GSW to the back during a hostile attack in Afghanistan, which resulted in complete motor and sensory deficit below the level of the injury at T9. The patient was dragged to safety, immediately evacuated to a local medical treatment facility, and then to the primary combat support hospital in Bagram. His neurological exam improved spontaneously in the first 24 hours, but he still had significant motor and sensory deficits (American Spinal Injury Association C-D-motor incomplete) [5]. His tetanus vaccination was current, antibiotic prophylaxis (cefazolin) was started, he remained on spinal precautions, and was evacuated expeditiously from the Afghan theater to Landstuhl Regional Medical Center for further evaluation and treatment. Upon arrival on post-injury day 2 , the patient had regained full lower extremity motor function but continued to complain of numbness in both lower extremities and very painful hyperesthesia in the T10-T12 dermatomes (subumbilical area) bilaterally. All neurological symptoms and pain were worsened with movement in bed. He was unable to reliably indicate great toe position bilaterally and had no vibration sense. Rectal exam was normal and reflexes were present. He was voiding spontaneously with good urinary control, but he had saddle pain and decreased sensation. The entry wound was a small punctate lesion in the left sub-scapular region. A computed tomography-scan of the thoracic spine demonstrated a metal fragment in the spinal canal at the T9 level with minimal comminution of the top of the left T10 lamina, indicating that the bullet entered into the canal at the T9-T10 interspace (Fig. 1). Magnetic resonance imaging was not possible because of the metal fragment.

The patient was thoroughly informed about the situation and counseled on the risks and benefits of surgical intervention; he opted to proceed with surgery. At surgery, the patient was placed in the prone position on a Jackson flat-top table with a Wilson frame. X-rays were used to localize the fragment at the T9 level. A midline incision was made from about T8 to T10, and a subperiosteal dissection was performed to expose the T9-T10 interspinous segment and T9 lamina. The bullet had caused a fracture of the inferior portion of the T9 lamina and the superior portion of the T10 lamina. A T9 laminectomy was then completed, to expose the traumatic durotomy from the bullet. The linear dural tear measured about $1 \mathrm{~cm}$ and was extended to a total of $2 \mathrm{~cm}$ to completely expose the bullet. The obvious metal bullet was identified lying on, but not in, the dorsal column of the spinal cord (Fig. 2A). The bullet was elevated, removed, and
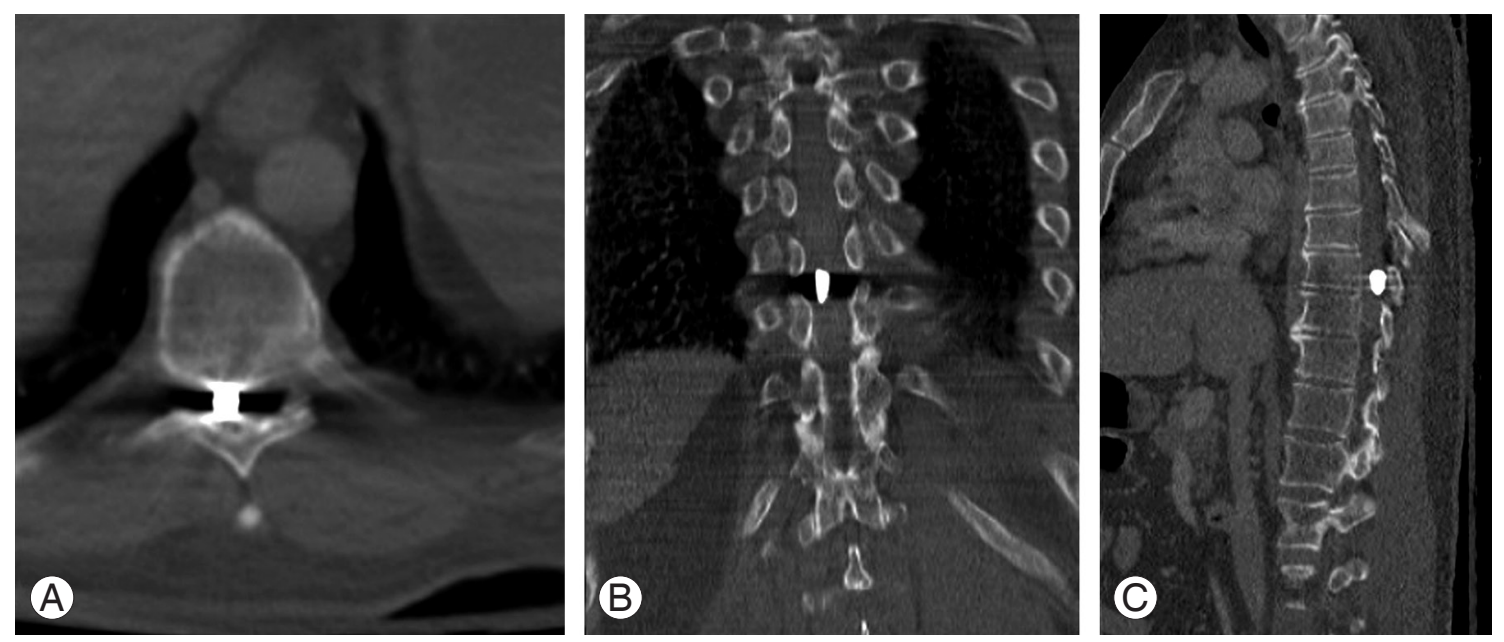

Fig. 1. Computed tomography scan of the spine shows the fragment in an intra-canicular position at the T9-10 level in the axial (A), coronal (B) and sagittal (C) planes. 
passed off the table (Fig. 2B). The durotomy was repaired primarily in a water-tight fashion and fibrin-glued (Evicel, Ethicon Inc., Somerville, NJ, USA) and the wound
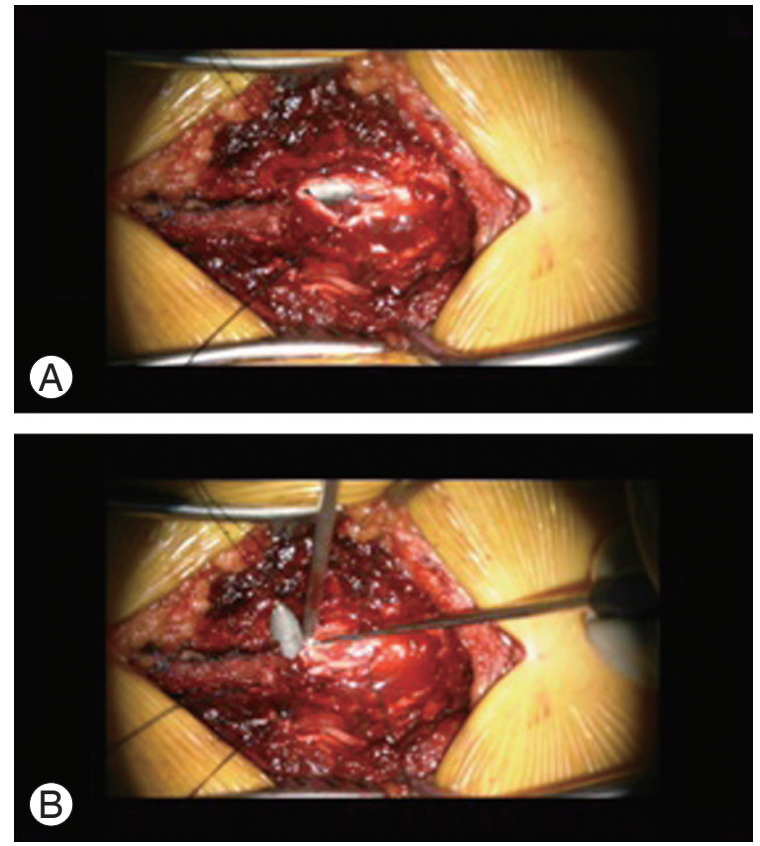

Fig. 2. Intraoperative microscope pictures showing the extended traumatic durotomy through the T9 laminectomy defect and the silver hue of the bullet lying on the dorsal column (A). (B) Shows the bullet being extracted gently. was closed in multiple anatomical layers. Intraoperative motor-evoked potentials were performed during the operation with no change from baseline.

In the immediate postoperative period, the wound healed with no evidence of cerebrospinal fluid (CSF) leakage or infection. The pain and increased neurological complaints, exacerbated by changes of position, resolved following the surgery. On examination, the motor function remained fully intact and sensation in the lower extremities improved to a protective level. Proprioception improved as well, while vibration sense remained absent. His very painful sub-umbilical hyperesthesia and saddle area dysthesias resolved to a persistent ache. He had a coordinated, slightly widened, tandem gait and was able to ambulate without assistive devices. In-line walking was compromised. Minimal further neurological improvement was seen in the ensuing 3 months. However, the patient is very satisfied with the outcome and his decision to have the fragment removed. He feels he is significantly better than prior to surgery and would elect the same treatment in the same situation. Further, he takes solace in the fact that a fragment inflicted in hostility was removed, thus obviating potential issues like migration, infection, and local toxic effects. Postoperative MRI of the spine (Fig. 3) revealed contusion of the spinal cord at the
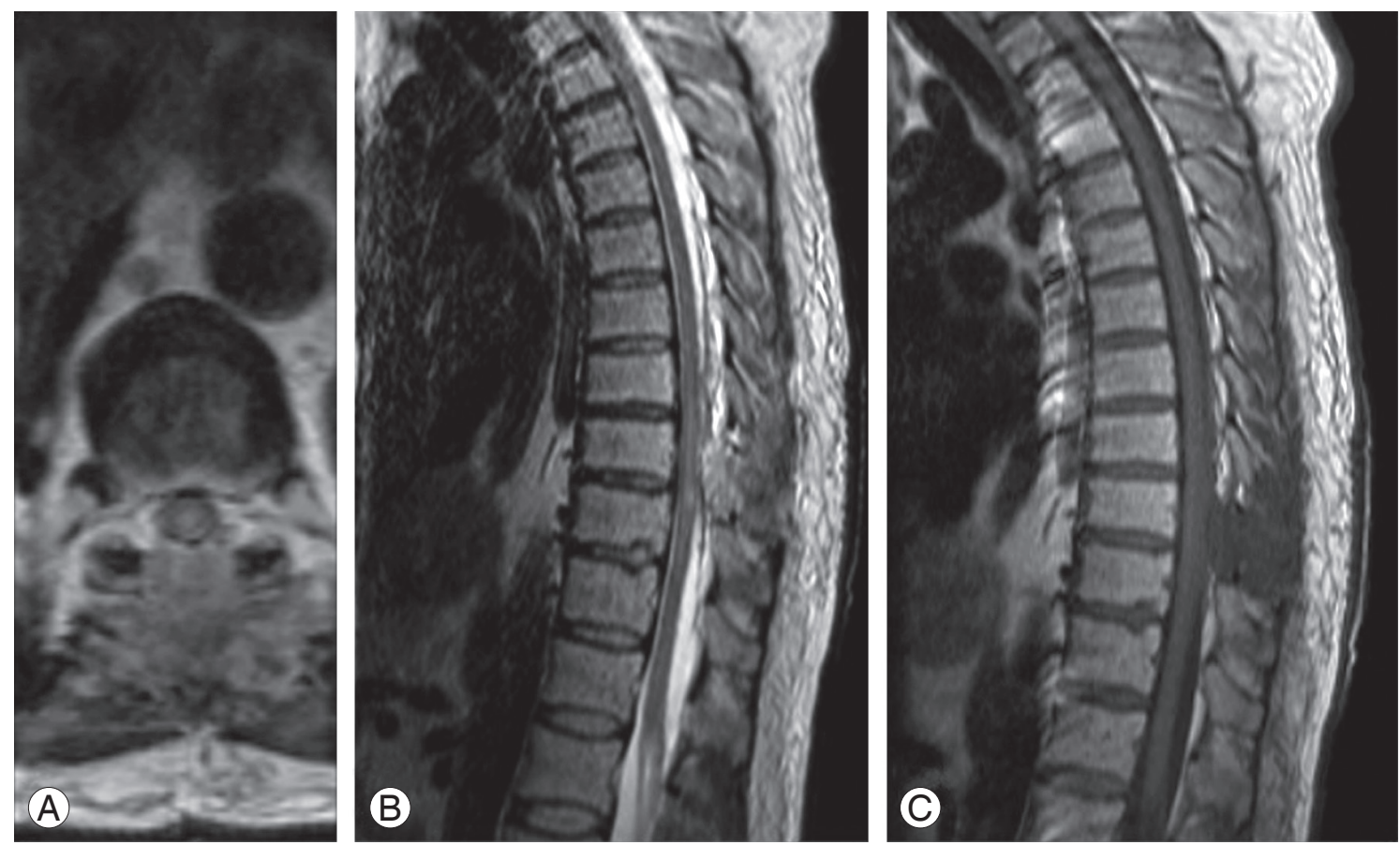

Fig. 3. Postoperative magnetic resonance imaging of the T-spine, shows a large (extending longitudinally about 2 levels [from lower-T8 to upper T10]) intramedullary T2 signal (A, B) without associated T1 signal (C) change consistent with spinal cord contusion and no obvious persistent cerebrospinal fluid leak or on-going spinal cord compression. 
T9 level, with no evidence of hemorrhage or transection.

Forensic evaluation of the extracted bullet confirmed it to be a steel armor-piercing 7.62-mm AK-47 round. The jacket had fractured off, suggesting that the bullet had hit a primary object prior to penetrating his spinal canal, which likely reduced the ballistic effect.

\section{Discussion}

According to Blair et al. [6], the incidence of spinal column injury was $5.45 \%$ of 10,979 combat casualties evacuated from theater from 2001 to 2009 , of which $17 \%$ had spinal cord injuries (SCIs) resulting in substantial disability. SCIs caused by explosions were complete in $18 \%$ of cases whereas those caused by GSWs were complete in $68 \%-84 \%$ of cases, illustrating the devastating force accompanying GSWs, versus penetrating projectiles from blast injuries [6-8]. In 1999, it was estimated by Cook et al. [9], based on 1994 data, that lifetime costs of GSWs were $\$ 2.3$ billion in the US, stating that SCIs accounted for a disproportionally large share of overall costs.

Regarding the wounding capacity of penetrating fragments, and in particular bullets, velocity at impact is the most important factor, because the velocity's impact on kinetic energy is squared. Other factors include the weight, size, form, and design (jacketed/non-jacketed) of the bullet [10]. Bullets damage tissues through direct injury, compressive stress or shockwaves, and temporary cavitation which are larger with more kinetic energy [11]. High-velocity GSWs to the spine are thus more likely to produce complete paraplegia, not only by direct trauma and compression to the spinal cord, but also indirectly through larger energy distribution with temporary cavitation and/or impairment of the vascular supply $[8,12]$.

There is uncertainty regarding the efficacy of surgery and long-term neurological outcomes for SCI following GSWs. Most studies agree, however, on the need for closing CSF fistulas and that acute surgical decompression is indicated when there are bone or missile fragments or soft tissue within the spinal canal in the setting of deteriorating neurological function [13-15]. Waters and Sie [15] and Waters and Adkins [16] determined that decompression and removal of bullets below the T12 level resulted in neurological improvement; however, at the thoracic and cervical levels, there was no significant effect on neurological outcome. The spinal cord is more susceptible to injury than the conus medullaris and cauda equina, which are composed in part of and solely of lower motor neurons with 'root recovery' potential $[4,14]$. In contrast to the findings of Waters and Sie [15] and Waters and Adkins [16], our patient, with a thoracic spinal cord GSW, improved after bullet removal.

Conservative treatment of foreign bodies within the spinal canal is associated with potential pitfalls such as local toxic effects and migration. In a study examining the effects of locally implanted bullet fragments in rabbits, it was found that after 9 months, especially copper intradural fragments caused extensive fibrosis and gliosis with tissue necrosis and loss of myelin and axons. Lead caused a similar but less severe reaction and aluminum was the most inert. No pathological effect on the spinal cord was seen with extradural fragments (copper, lead, or aluminum) [17]. Tungsten alloys, used by many countries as an alternative to lead-containing materials in recent years, have been associated with the potential to develop highgrade rhabdomyosarcomas [18]. Furthermore there have been cases described with late-onset neurological deficit due to reactive changes around intraspinal bullets [19]. Another potential problem is the possible migration of the foreign object within the spinal canal. Although this is thought to be limited to below the T10 level, there have been cases reported of migration in the thoracic level and higher $[20,21]$. The bullet in our case was lying freely on the cord in the subarachoid space and was easily lifted from the area at the time of surgery.

GSWs perforating the alimentary tract, especially the colon, before lodging in the spine or spinal canal are associated with both wound and spine infections [22]. With high-velocity bullets, materials such as field clothing and skin fragments may be carried or sucked into the wound, creating significant deep contamination and the potential for infection [23]. Recommendations for antibiotic prophylaxis are dictated by the presumed or confirmed path of the bullet [24]. When the bullet only traverses soft tissue, a first-generation cephalosporin (e.g., cefazolin) for 2-3 days is sufficient. When the bullet traverses a viscous organ, broader range (e.g., third-generation cephalosporin) and longer duration (7-14 days) prophylaxis is recommended. In all cases, tetanus status should be confirmed to be current, and when it is not or unknown, the patient should be vaccinated. Following these recommendations, our patient remained free of infection.

Timing in performing surgery seems to be important too. In a recent study by Klimo et al. [11], guidelines 
were established based on an extensive review of both military and civilian literature. They concluded that with incomplete neurological deficit, when there is evidence of significant spinal canal compromise, surgery should be performed as soon as possible, ideally within $24-48$ hours. We fully explained the situation to the patient, empowered him to elect surgical intervention, and proceeded with surgery within this time frame, and removed the bullet successfully, leading to significant improvement of his incomplete posterior cord syndrome.

High-velocity (and high-caliber) bullets penetrating into the spine have enormous destructive force, most often resulting in permanent complete neurological deficits. Despite a GSW to the spinal cord, our patient achieved significant improvement. This patient's recovery lends support to operative extraction of an (combat-related) intradural foreign body in the setting of incomplete SCI produced by GSW. This case does not speak to the more common setting of a complete spinal cord injury with no evidence of spontaneous improvement, in which case there is essentially no likelihood of significant neurological improvement. The efficacy and timing of surgery in the setting of complete injuries remains controversial and is not informed by this case.

\section{Conflict of Interest}

No potential conflict of interest relevant to this article was reported.

\section{References}

1. Belmont PJ Jr, McCriskin BJ, Sieg RN, Burks R, Schoenfeld AJ. Combat wounds in Iraq and Afghanistan from 2005 to 2009. J Trauma Acute Care Surg 2012;73:3-12.

2. Lee KH, Lin JS, Pallatroni HF, Ball PA. An unusual case of penetrating injury to the spine resulting in cauda equina syndrome: case presentation and a review of the literature. Spine (Phila Pa 1976) 2007;32:E290-3.

3. Spaic M, Kostic V, Milosavljevic I. Gunshot wound to the spine without neurological loss: a case report. Indian J Neurotrauma;9:52-5.

4. Harrop JS, Naroji S, Maltenfort MG, et al. Neurologic improvement after thoracic, thoracolumbar, and lumbar spinal cord (conus medullaris) injuries. Spine
(Phila Pa 1976) 2011;36:21-5.

5. Marino RJ, Barros T, Biering-Sorensen F, et al. International standards for neurological classification of spinal cord injury. J Spinal Cord Med 2003;26 Suppl 1:S50-6.

6. Blair JA, Patzkowski JC, Schoenfeld AJ, et al. Spinal column injuries among Americans in the global war on terrorism. J Bone Joint Surg Am 2012;94:e135(19).

7. Blair JA, Possley DR, Petfield JL, et al. Military penetrating spine injuries compared with blunt. Spine J 2012;12:762-8.

8. Alaca R, Yilmaz B, Goktepe AS, Yazicioglu K, Gunduz S. Military gunshot wound-induced spinal cord injuries. Mil Med 2002;167:926-8.

9. Cook PJ, Lawrence BA, Ludwig J, Miller TR. The medical costs of gunshot injuries in the United States. JAMA 1999;282:447-54.

10. DeMuth WE Jr. Bullet velocity and design as determinants of wounding capability: an experimental study. J Trauma 1966;6:222-32.

11. Klimo P Jr, Ragel BT, Rosner M, Gluf W, McCafferty R. Can surgery improve neurological function in penetrating spinal injury? A review of the military and civilian literature and treatment recommendations for military neurosurgeons. Neurosurg Focus 2010;28:E4.

12. Mirovsky Y, Shalmon E, Blankstein A, Halperin N. Complete paraplegia following gunshot injury without direct trauma to the cord. Spine (Phila Pa 1976) 2005;30:2436-8.

13. Jallo GI. Neurosurgical management of penetrating spinal injury. Surg Neurol 1997;47:328-30.

14. Kitchel SH. Current treatment of gunshot wounds to the spine. Clin Orthop Relat Res 2003;(408):115-9.

15. Waters RL, Sie IH. Spinal cord injuries from gunshot wounds to the spine. Clin Orthop Relat Res 2003;(408):120-5.

16. Waters RL, Adkins RH. The effects of removal of bullet fragments retained in the spinal canal. A collaborative study by the National Spinal Cord Injury Model Systems. Spine (Phila Pa 1976) 1991;16:934-9.

17. Tindel NL, Marcillo AE, Tay BK, Bunge RP, Eismont FJ. The effect of surgically implanted bullet fragments on the spinal cord in a rabbit model. J Bone Joint Surg Am 2001;83:884-90.

18. Kalinich JF, Emond CA, Dalton TK, et al. Embedded 
weapons-grade tungsten alloy shrapnel rapidly induces metastatic high-grade rhabdomyosarcomas in F344 rats. Environ Health Perspect 2005;113:729-34.

19. Ajmal S, Enam SA, Shamim MS. Neurogenic claudication and radiculopathy as delayed presentations of retained spinal bullet. Spine J 2009;9:e5-8.

20. Oktem IS, Selcuklu A, Kurtsoy A, Kavuncu IA, Pasaoglu A. Migration of bullet in the spinal canal: a case report. Surg Neurol 1995;44:548-50.

21. Cheng JS, Richardson RM, Gean AD, Stiver SI. Delayed acute spinal cord injury following intra- cranial gunshot trauma: case report. J Neurosurg 2012;116:921-5.

22. Quigley KJ, Place HM. The role of debridement and antibiotics in gunshot wounds to the spine. J Trauma 2006;60:814-9.

23. Cooper GJ, Ryan JM. Interaction of penetrating missiles with tissues: some common misapprehensions and implications for wound management. Br J Surg 1990;77:606-10.

24. Bono CM, Heary RF. Gunshot wounds to the spine. Spine J 2004;4:230-40. 